\title{
Coordinated Action in a Heterogeneous Rescue Team
}

\author{
Fares Alnajar, Hanne Nijhuis, and Arnoud Visser \\ Intelligent Systems Laboratory Amsterdam, Universiteit van Amsterdam, \\ Science Park 107, NL 1098 XG Amsterdam, The Netherlands \\ f.alnajar@student.uva.nl, h.nijhuis@student.uva.nl, a.visser@uva.nl
}

\begin{abstract}
In this paper we describe a new approach to make use of a heterogeneous robot team for the RoboCup Rescue League Virtual Robot competition. We will demonstrate coordinated action between a flying and a ground robot. The flying robot is used for fast exploration and allows the operator to find the places where victims are present in the environment. Due to the fast aggregation of the location error in the flying robot no precise location of the victim is known. It is the task of the ground robot to autonomously go the point of interest and to get an accurate location of the victim, which can be used by human rescue workers to save the victim. The benefit of this approach is demonstrated in a small number of experiments. By integrating the abilities of the two robots the team's performance is improved.
\end{abstract}

\section{Introduction}

Since long it has been indicated that a heterogeneous robot team should have operational benefits [1]. For Urban Search and Rescue operations, the benefits seem even more promising [2]. Many teams 34 have indicated the possibility of heterogeneous team consisting of an aerial and ground robot, but actual demonstrations are sparse [5]. Here, the benefit of coordinated action between a teleoperated robot and a semi-autonomous ground-robot is demonstrated.

\subsection{Relevance}

In situations where a disaster like an earthquake has occurred, searching for survivors in the area could be dangerous due to (partly) collapsed buildings that are unstable. It could also be difficult for humans to search in such a collapsed building if the available room is too small to crawl through. In such situations robots could be deployed to search the area and hopefully supply some useful information on the location and status of possible survivors. These robots could be operated by humans (by remote control), but if they are able to explore (semi) autonomously one could deploy a whole team of robots simultaneously to cover a bigger area. To investigate the possibilities of multiple robots in these situations, the Virtual Robot competition of the RoboCup Rescue League was introduced [6].

J. Baltes et al. (Eds.): RoboCup 2009, LNAI 5949, pp. 1-10, 2010.

(C) Springer-Verlag Berlin Heidelberg 2010 
Because deploying actual robots in actual disaster-situations is a very complex task, the virtual robots competition is done in a simulated world. Simulation should not replace experiments with real robots, but a simulated world makes complex experiments reproducible and controllable. This provides the opportunity for rapid development by focusing on only a relevant aspect. Researchers can use this simulated world as rapid development environment, and can explore the design space for behaviors and co-operation between multiple robots before they are validated on real platforms.

\subsection{USARSim}

Since 2006 there have been annual world competitions in a simulation environment called USARSim. It's based on the Unreal Tournament 2 engine [7] and provides the ability to have robots operate in a 3D world with the laws of physics (like gravity) already implemented.

For our research the CompWorldDay 1 mant is used as 3D map. This map supplies a large outdoor- as well as indoor-environment (an overview of the outdoor area we operate in can be seen in Figure 1(c). Many obstacles like cars, buildings and construction are present, but the sky is fairly empty. This will give the aerial robot the opportunity to fly around without excessive need for 'obstacle avoidance'.

Robots are recreated in the virtual world, based on real machines that are

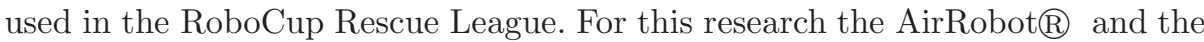
Pioneer 2-AT (P2AT) were used, as are depicted in figure 1(a) and 1(b)

\subsection{Performance Metrics}

To be able to compare the performance of the participating teams some metrics were defined. The initial 2006 metrics were specified with the following formula:

$$
S=\frac{V_{I D} \times 10+V_{S T} \times 10+V_{L O} \times 10+t \times M+E \times 50-C \times 5+B}{(1+H)^{2}}
$$

Where $V_{I D}$ is the number of victims identified, $V_{S T}$ is the number of victims for which a status was reported, $V_{L O}$ is the number of properly localized victims, $B$ is an optional amount of bonus points rewarded by a referee for additional information on victims, $t$ is a scaling factor for the accuracy of the map, $M$ is the points assigned by a referee for the quality of the map, $E$ is the points assigned by a referee for the exploration efforts, $C$ the collisions between a robot and a victim and $H$ the number of operators.

Over the years these metrics have changed [8] into the latest 2009 metrics:

$$
S=\frac{E * 50+M * 50+V * 50}{(H)^{2}}
$$

\footnotetext{
${ }^{1}$ Available for download on: http://downloads.sourceforge.net/usarsim/
} 


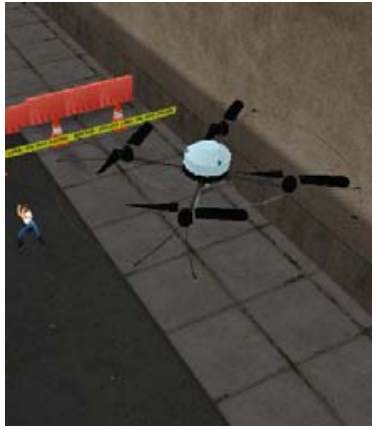

(a) The AirRobot

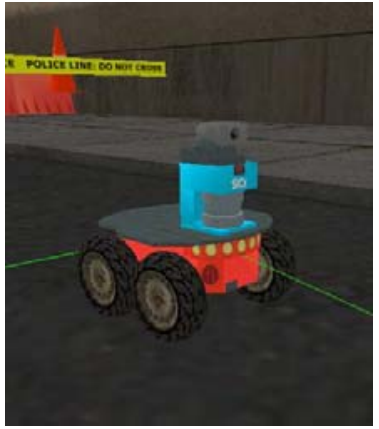

(b) The P2AT ground robot

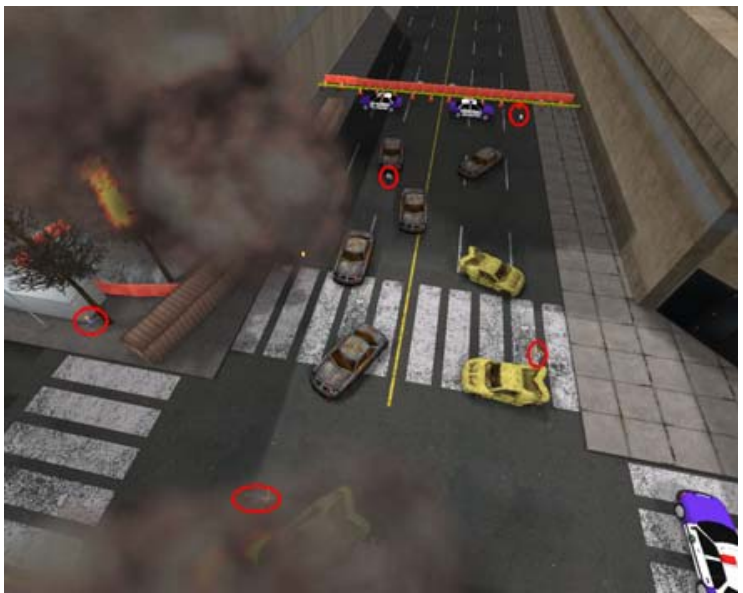

(c) Outdoor area of CompWorldDay1, as soon through the camera of the AirRobot. Victim locations are indicated by red circles.

Fig. 1. Images from the USARSim environment

where $V$ is an aggregate number indicating the effectiveness of victim detection and $H \geq 1$ is guaranteed. Part of $V$ is the accuracy of the localization of the victim; if the victim is reported more than 2.5 meters from its actual location it is counted as false alarm with a corresponding penalty.

Although the AirRobot can be used to detect victims, initial tests indicated that the accuracy of localization of the AirRobot drops fast after a few turns. Although not impossible, building a map on visual clues alone is quite challenging 9. Due to the limitations of the AirRobot, the performance of heterogeneous team will be based on the map of the ground-robot alone.

\subsection{Related Work}

Experiments with a heterogeneous team in rescue applications have been tried before. The 2nd place of the 2008 Virtual Robot competition was actually 
rewarded to a heterogeneous team consisting of one aerial and one ground robot [5]. Note that both robots were manually operated. Our approach incorporates semi-autonomous behavior as explained in section 3.1. Semi-autonomy by following an online planned path is demonstrated by many teams in the competition (e.g. 431011]). To coordinate the actions of multiple robots by dropping RFID tags is inspired by the approach introduced by the Freiburg team [12]. The Freiburg robots avoided places where RFID tags were dropped, to optimize the exploration efforts $E$. Our ground robot actively searches for RFID tags, to optimize the victim detection $V$. In both approaches the location of the RFID tags is used to ensure that all robots use the same global locations.

\subsection{Outline}

In the following section we will explain the approach we took on showing improvements by using a heterogeneous team. Next we will cover the methods we used to implement this approach in section 3. We will discuss the experiments and their results in section 4 to round of with a discussion and a conclusion.

\section{Approaches}

We discuss several approaches to make the heterogeneous team operate (semi) autonomously. For the moment our approach is tested on outdoor situations only.

\subsection{Exploration}

The main advantages of the AirRobot are its speed and its ability to move at such height that obstacles are relatively sparse (you can fly over the obstacles instead of driving around them). This would make the AirRobot ideal for exploring a large area quickly. Furthermore the ability to explore from a high position provides a higher probability of having an unobstructed view on victims.

Unfortunately the pay-load of the robot is very limited, so in real-life situations we can not supply the robot with heavy gear. This limits not only the amount and type of sensors the robot could carry, but also the computational power available on board. For this research the payload is limited to a camera, a victim-sensor, a RFID dispenser and an inertia navigation system.

\subsection{Victim Localization}

Since the main goal of the Rescue League is to find victims in a disaster-area we want to use the AirRobot to search for them, because this can be done much faster from the sky than by a slow ground robot. The AirRobot uses its inertia navigation system (acceleration sensor) and therefore rapidly accumulates an error on the estimation of its location. The threshold on accurate victim localization is so sharp that GPS is also no option. This means that a victim report of the AirRobot could not be trusted, and should be verified by a ground robot. 


\subsection{Sharing Knowledge}

Each member of the team (the ground robot and the aerial one) makes an estimation of its position. Both robots have an inertial navigation system, which gives a relative position estimate. This relative position estimate is used by the ground robot as initial estimate for a SLAM routine [13, which returns an absolute location estimate by comparison of the current range measurements against previous measurements aggregated on a map.

To unify the location estimation of the two robots, the estimations of a specific point by the two robots are computed and used to find out the shift the aerial robot makes with respect to the ground one.

There are two ways to achieve this 'unification'. In the first one, the ground robot computes this shift and sends the 'correction' to the aerial robot for the next estimations. But this method requires a consistent connection with the aerial robot which is not always available in the disaster situations, and requires the two robots to be close to each other. The second way is by storing the shift 'correction' at the ground robot as list for every victim/RFID tag it has reached. The ground robot uses this list to correct the position it is currently heading. When the aerial robot comes back again into communication range the ground robot transmit the whole list to allow the aerial to correct its traveled path as a sort of post-processing, as described in section 3.2 .

\subsection{Cooperation}

The two robots of our team have clearly different roles. The aerial robot is used for exploring the environment and searching for victims. This robot is teleoperated. The aerial robot sends the approximated locations of the victims to the ground one, which is also equipped with a victim sensor. So as the ground robot gets close to the victim, it can detect its exact position, and make an accurate victim report. The positions sent by the aerial robot are imprecise, and only used guide the ground robot to the neighborhood of the victim. The ground robot makes further investigation for the victim in that neighborhood (the victim sensor has a range of 6 meters). There is no guarantee that the absolute position error of the AirRobot is less than 6 meters, but because the ground robot maintains a gradual 'correction' of the aerial robot localization, this strategy is only sensitive for the accumulation of the error between two victim locations.

\section{Methods Used}

In this section we explain the details of the semi-autonomous following-behavior of the ground robot to navigate the environment. Also we discuss how the two robots calculate the correction vector for the localization estimate of the aerial robot. 


\subsection{Following}

The follow-behavior (as used by the ground robot) is a collection of 'motions' and the rules to switch from one motion to another. The switch between two motions depends on observations of certain events in the local environment around the robot (e.g. presence of obstacles, victims, and/or other teammates). A switch should indicate when a motion is not longer adequate for this situation. This behavior is built on the motions used for the fully autonomous exploration [14. The motion contains the reactive schemes that directly couple sensor measurements to steering commands which navigate the environment. In each moment only one motion is active, and the robot behavior switches from one motion to another, depending on the robots situation. Here we give a description of these motions.

Following: This is the default motion of the follow-behavior, in which the robot waits for the position of the next target (the victim positions). When it receives a target-position, it plans online a path from its current position to the target, based on the occupancy grid map build so far. The pathplanning in this experiment is based on a breath-first algorithm, but also an $\mathrm{A}^{*}$-algorithm is available. After the robot drove a certain distance (4 meters) it re-plans the path again on the new occupancy grid map, to incorporate information about the environment previously not visible.

Avoid Teammate: This motion is called when there is potential risk of collision between two robots (when the AirRobot is still on the ground and the distance between them is less than 1 meter). There are four states the robot can have while trying to avoid a teammate:

- The teammate is facing the robot from the front side: in this case the robot should turn right or left to get out of the way.

- The teammate facing the robot from the back side: the same as the previous state.

- The teammate is in front of the robot but not facing it: the robot waits till the teammate move a way

- The teammate is behind the robot but not facing it: the robot keeps its normal behavior (return the control to the 'following' motion).

Avoid Victim: This motion is used when the robot gets closer to the victim less than 1 meter. After detecting the closest part of the victim, the motion keeps the robot away of the victim. When the robot is far (more than 2 meters), the control is returned to the following motion.

As we can see the flowing of control from one motion and another depends on the situation of the robot, this is depicted in figure 2.

\subsection{Updating Location Estimation Shift}

Essential in our approach is calculation the difference in location estimation between the team members. Our method is by based on dropping RFID tags, 


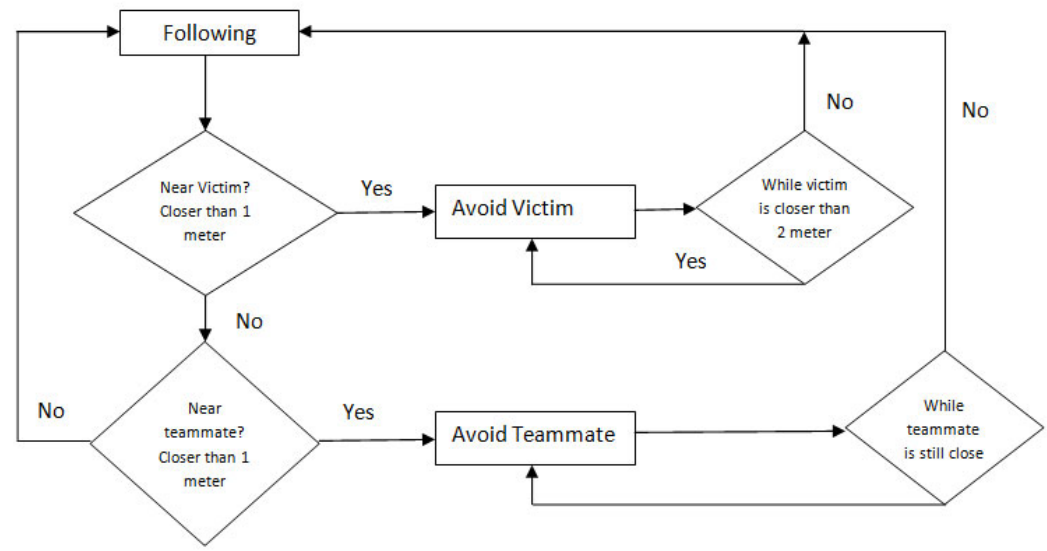

Fig. 2. Diagram of the follow-behavior

equivalent with the method used by 12 , but now to find target points back. An RFID tag is small and cheap radio wave responder, which is often used for tracking and identification of logistic goods. These tags can be used as 'smart dust'; at some points the AirRobot stores its current location estimate in a RFID tag and drops this tag. When the ground robot comes close enough to this RFID tag, it can read the content the tag, and its current absolute location estimate and the read position as read from the tag (which was the location previously estimated by the aerial robot). Based on this comparison the new shift vector is calculated.

\section{Experiments and Results}

We tested our heterogeneous team on outdoor environment. We used the "P2AT"model for the ground robot. The ground robot visits the places reported by the aerial robot and detect the victim locations accurately. At end of the test run, there were even some victims that the ground robot didn't have time to visit and detect their exact position.

To map illustrated in Fig. 3 is the result of a short flight of 8 minutes. Full competition runs can last 20 to 40 minutes. In this experiment three tags are dropped by the AirRobot, two near a victim, and one as intermediate point. Victims are reported as small red crosses. As can be seen, for each victim two crosses are given: the location as estimate by the AirRobot and the position as estimated by the P2AT. The difference is between the estimates is nearly 3 meters, above the threshold of the competition. Only by the ground-robot the victims are reported at the right location. As supplementary material also a video is available 2 , from the view of the ground-robot. In the video can be seen that the ground-robot is constantly reading RFID tags. Most of them are the

\footnotetext{
2 http://www.science.uva.nl/ arnoud/publications/Alnajar_CoordinatedAction.wmv
} 


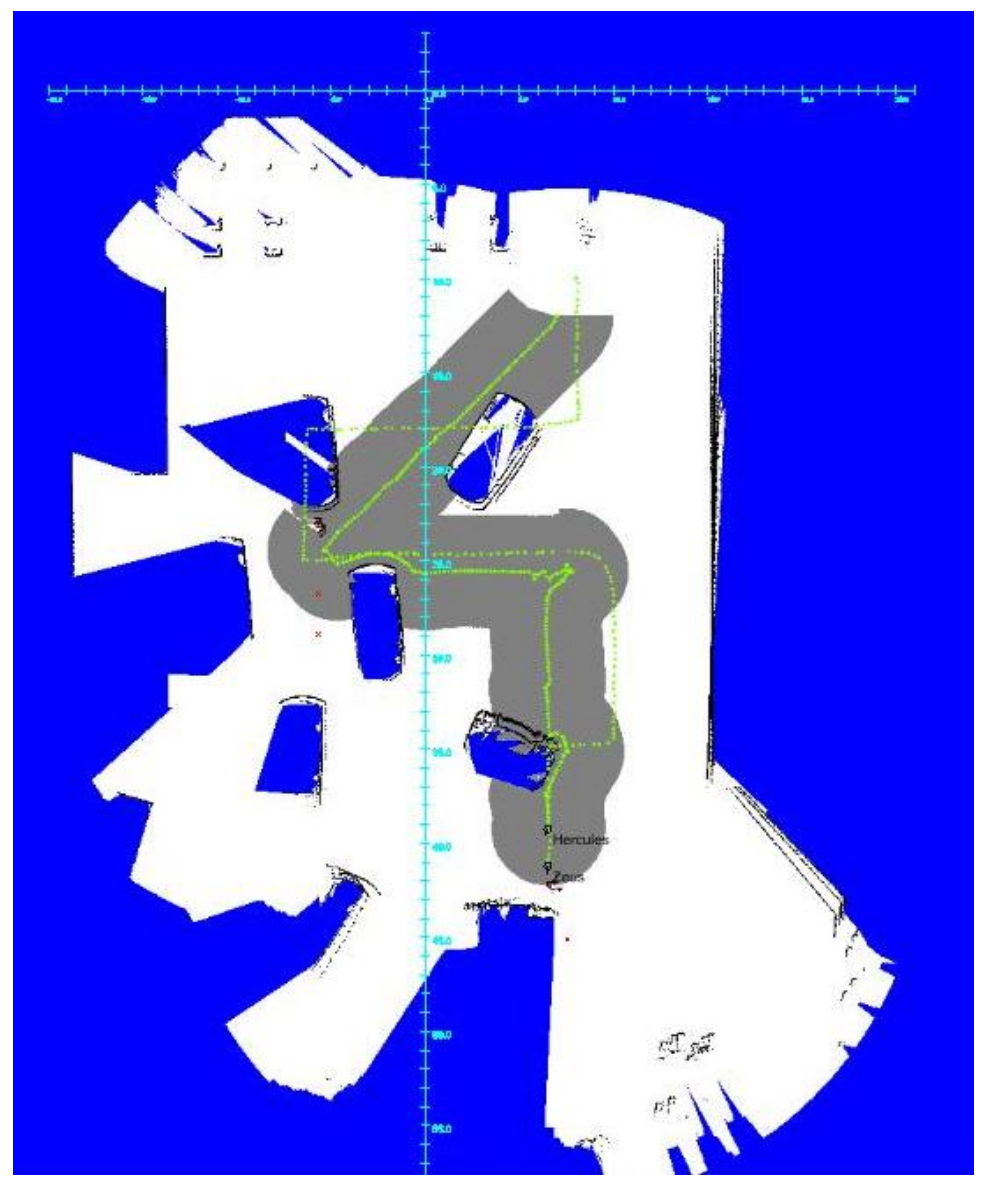

Fig. 3. Map generated by the ground robot in the outdoor area of CompWorldDay1

RFID tags with only an ID always present in the 2006 competition world 3 . Only near the male and female victim RFID tags are dropped by the AirRobot.

\section{Discussion}

Our team works well in the outdoor environment, but care must be taken when testing the team in indoor environments. The exploration of the aerial robot becomes very restricted due to the small free space and the many obstacles around the robot. As long as the AirRobot has no obstacle sensors, it has to remain in the area already explored by the ground robots. When the ground robots are equipped with sophisticated range scanner, this is not a severe restriction,

${ }^{3}$ Used at that time to estimate the accuracy of the maps, currently replaced by georeferenced maps. 
because this range scanner can have a range of 80 meters. The remaining danger are obstacles which are not straight (as most buildings), but have a larger volume at the flight height of the AirRobot than at the scan-height of the ground-robots (as most trees). These obstacles can only be avoided with obstacle sensors on board of the robot. Due to the limited payload of the AirRobot such obstacle sensors have to be carefully chosen. A possible solution could be obstacle detection based on visual clues.

Another topic of future work is to achieving the relative position estimation update via visual tracking. Currently the relative position between the two robots is quickly lost, something that is partly corrected when a RFID tag is found. If the ground-robot could visually track the AirRobot, a high frequency update of this correction could be made (when an accurate height position is available). Using a bright color for the AirRobot (e.g. orange) can make the tracking easier.

Another possibility is extending the team size further. Many questions could be studied: How to divide the work of the ground robots (going to the victims)? What's the difficulty in adding more than one flying robot? What is the optimal ratio between the ground robots and the aerial ones? How can teams of aerial and ground robots automatically be formed?

\section{Conclusion}

In this paper a new coordinate action between an AirRobot and a ground robot is described. The two robots coordinate their behavior partly by communicating target points and partly through the environment by dropping and finding RFID tags. Each robot has its own role. The AirRobot is used for fast and course exploration, while the ground robot automatically inspects the regions of interest and needs no further attention of the operator. Together the heterogeneous team demonstrates an attractive way to perform a rescue mission.

\section{References}

1. Jennings, J., Whelan, G., Evans, W.: Cooperative search and rescue with a team of mobile robots. In: Proc. of the IEEE Int. Conf. on Advanced Robotics (ICAR), pp. 193-200 (1997)

2. Murphy, R., Casper, J., Micire, M., Hyams, J.: Mixed-initiative Control of Multiple Heterogeneous Robots for USAR. Technical Report CRASAR-TR2000-11, Center for Robot Assisted Search \& Rescue, University of South Florida, Tampa, FL (2000)

3. Pfingsthorn, M., Rathnam, R., Stoyanov, T., Nevatia, Y., Ambrus, R., Birk, A.: Jacobs Virtual Robot 2008 Team - Jacobs University Bremen, Germany. In: Proceedings CD of the 12th RoboCup Symposium (2008)

4. Calisi, D., Randelli, G., Valero, A., Iocchi, L., Nardi, D.: SPQR Rescue Virtual Robots Team Description Paper. In: Proceedings CD of the 12th RoboCup Symposium (2008)

5. Balaguer, B., Carpin, S.: UC Mercenary Team Description Paper: RoboCup 2008 Virtual Robot Rescue Simulation League. In: Proceedings CD of the 12th RoboCup Symposium (2008) 
6. Nourbakhsh, I.R., Sycara, K., Koes, M., Young, M., Lewis, M., Burion, S.: HumanRobot Teaming for Search and Rescue. Pervasive Computing, 72-78 (2005)

7. Balakirsky, S., Scrapper, C., Carpin, S., Lewis, M.: USARSim: Providing a Framework for Multi-robot Performance Evaluation. In: Proc. of the Performance Metrics for Intelligent Systems (PerMIS) Workshop, pp. 98-102 (2006)

8. Balakirsky, S., Scrapper, C., Carpin, S.: The Evolution of Performance Metrics in the RoboCup Rescue Virtual Robot Competition. In: Proc. of the Performance Metrics for Intelligent Systems (PerMIS) Workshop, pp. 91-96 (2007)

9. Booij, O., Terwijn, B., Zivkovic, Z., Kröse, B.: Navigation using an appearance based topological map. In: Proceedings of the International Conference on Robotics and Automation (ICRA 2007), Roma, Italy, pp. 3927-3932 (2007)

10. Velagapudi, P., Kwak, J., Scerri, P., Lewis, M., Sycara, K.: Robocup Rescue Virtual Robots Team STEEL (USA) - MrCS - The Multirobot Control System. In: Proceedings CD of the 12th RoboCup Symposium (2008)

11. Visser, A., Schmits, T., Roebert, S., de Hoog, J.: Amsterdam Oxford Joint Rescue Forces - Team Description Paper - Virtual Robot competition - Rescue Simulation League - RoboCup 2008. In: Proceedings CD of the 12th RoboCup Symposium (2008)

12. Ziparo, V.A., Kleiner, A., Marchetti, L., Farinelli, A., Nardi, D.: Cooperative Exploration for USAR Robots with Indirect Communication. In: Proc. of 6th IFAC Symposium on Intelligent Autonomous Vehicles, IAV 2007 (2007)

13. Pfingsthorn, M., Slamet, B.A., Visser, A.: A Scalable Hybrid Multi-Robot SLAM Method for Highly Detailed Maps. In: Visser, U., Ribeiro, F., Ohashi, T., Dellaert, F. (eds.) RoboCup 2007: Robot Soccer World Cup XI. LNCS (LNAI), vol. 5001, pp. 457-464. Springer, Heidelberg (2008)

14. Visser, A., Slamet, B.A.: Balancing the Information Gain Against the Movement Cost for Multi-robot Frontier Exploration. In: Bruyninckx, H., Přeučil, L., Kulich, M. (eds.) European Robotics Symposium 2008. Springer Tracts in Advanced Robotics, vol. 44, pp. 43-52. Springer, Heidelberg (2008) 